\title{
ON THE SPECIAL VALUES OF ARTIN L-FUNCTIONS FOR DIHEDRAL EXTENSIONS
}

\author{
YUTAKA KONOMI
}

\begin{abstract}
We study special values of Artin $L$-functions for dihedral extensions at negative integers. We give a relation between these values and orders of the $\chi$-parts of certain étale cohomology groups.
\end{abstract}

Keywords: étale cohomology, $K$-group, class number, Iwasawa theory, Artin $L$-function.

\section{Introduction and the main result}

Let $p$ and $l$ be distinct odd primes. We denote by $D_{2 l}$ the dihedral group of order $2 l$. Let $L^{+}$be a dihedral extension over a number field $F^{+}$of degree $2 l$. Suppose that both $L^{+}$and $F^{+}$are totally real. For a totally positive algebraic number $r \in F^{+}$, let $L=L^{+}(\sqrt{-r})$ and $F=F^{+}(\sqrt{-r})$. Let $O_{L}$ be the integer ring of $L$. Let $\chi$ be a character of $\operatorname{Gal}\left(L / F^{+}\right)$. Denote by $\mathscr{L}\left(L / F^{+}, \chi, s\right)$ the Artin $L$-function attached to $\chi$ and put $d_{\chi}=\left[\mathbb{Z}_{p}[\operatorname{Im}(\chi)]: \mathbb{Z}_{p}\right]$. We say that $\chi$ is even if it is the inflation of a character of $\operatorname{Gal}\left(L^{+} / F^{+}\right)$, while odd if it is the product of an even character with the inflation of the non-trivial character of $\operatorname{Gal}\left(F / F^{+}\right)$. Moreover, $a \sim_{p} b$ signifies that $a$ and $b$ are two $p$-adic numbers with the same valuation. Let $H_{\text {êt }}^{i}\left(\operatorname{Spec} O_{L}[1 / p], \mathbb{Z}_{p}(n)\right)$ be the étale cohomology group, which we will simply denote by $H^{i}\left(O_{L}^{\prime}, \mathbb{Z}_{p}(n)\right)$. The main result of this paper is the following theorem.

Theorem 1.1. Let $n \geqslant 2$ be an integer and $\chi$ an irreducible character of $\operatorname{Gal}\left(L / F^{+}\right)$. Assume that $\chi$ is even if $n$ is even and $\chi$ is odd if $n$ is odd. Then

$$
\mathscr{L}\left(L / F^{+}, \bar{\chi}, 1-n\right)^{\chi(1) d_{\chi}} \sim_{p} \frac{\# H^{2}\left(O_{L}^{\prime}, \mathbb{Z}_{p}(n)\right)^{\chi}}{\# H^{1}\left(O_{L}^{\prime}, \mathbb{Z}_{p}(n)\right)^{\chi}},
$$

where $H^{i}\left(O_{L}^{\prime}, \mathbb{Z}_{p}(n)\right)^{\chi}$ means the $\chi$-part of $H^{i}\left(O_{L}^{\prime}, \mathbb{Z}_{p}(n)\right)$. 
The definition of $\chi$-part will be given in Section 2. Theorem 1.1 is close to the following known result for an abelian extension, which will be used by our proof.

Theorem 1.2 ([3], p. 707). Let $n \geqslant 2$ be an integer and $L / K$ a totally complex abelian extension of the totally real base field $K$ of degree prime to $p$. Let $\chi$ be a character of $\mathrm{Gal}(L / K)$, such that $\chi(-1)=(-1)^{n}$, and view $\chi$ as a $p$-adic character. Then

$$
\mathscr{L}\left(L / K, \chi^{-1}, 1-n\right)^{d_{\chi}} \sim_{p} \frac{\# H^{2}\left(O_{L}^{\prime}, \mathbb{Z}_{p}(n)\right)^{\chi}}{\# H^{1}\left(O_{L}^{\prime}, \mathbb{Z}_{p}(n)\right)^{\chi}} .
$$

Now, we can interpret Theorem 1.1 in terms of $K$-groups. For $n \geqslant 2$, it is seen that the $p$-adic Chern maps

$$
K_{2 n-i}\left(O_{L}\right) \otimes_{\mathbb{Z}} \mathbb{Z}_{p} \longrightarrow H^{i}\left(O_{L}^{\prime}, \mathbb{Z}_{p}(n)\right) \quad(i=1,2)
$$

are isomorphisms, which is known as the Quillen-Lichtenbaum conjecture (cf. [7], [8]). Consequently, Theorem 1.1 gives the relation

$$
\mathscr{L}\left(L / F^{+}, \bar{\chi}, 1-n\right)^{\chi(1) d_{\chi}} \sim_{p} \frac{\# K_{2 n-2}\left(O_{L}\right)_{\text {tors }}^{\chi}}{\# K_{2 n-1}\left(O_{L}\right)_{\text {tors }}^{\chi}}
$$

for $\chi$ with the same parity of $n \geqslant 2$. Further, we add the fact that (1.1) is essentially valid for $n=1$, by

$$
K_{0}\left(O_{L}\right) \simeq \mathbb{Z} \oplus \mathrm{Cl}_{L}, \quad K_{1}\left(O_{L}\right) \simeq O_{L}^{\times}
$$

and the main theorem of [4] (p. 1063). Here, $\mathrm{Cl}_{L}$ denotes the ideal class group of $L$.

\section{Proof of the main theorem}

Let $D_{2 l}=\langle a, b\rangle$ with $a^{l}=b^{2}=1$ and $b a b^{-1}=a^{-1}$. It is known that $D_{2 l}$ has the two one-dimensional representations and the $(l-1) / 2$ irreducible two-dimensional representations. The character table is as follows:

\begin{tabular}{l|ccr} 
& $1_{D_{2 l}}$ & $a^{i}\left(1 \leqslant i \leqslant \frac{l-1}{2}\right)$ & $b$ \\
\hline$\varepsilon$ & 1 & 1 & 1 \\
$\eta$ & 1 & 1 & -1 \\
$\chi_{k}\left(1 \leqslant k \leqslant \frac{l-1}{2}\right)$ & 2 & $\zeta_{l}^{i k}+\zeta_{l}^{-i k}$ & 0
\end{tabular}

where $\zeta_{l}=\exp (2 \pi \sqrt{-1} / l)$.

Take $\sigma \in \operatorname{Hom}\left(\langle a\rangle, \overline{\mathbb{Q}}^{\times}\right)$satisfying $\sigma(a)=\zeta_{l}$, and write $\sigma_{i}=\sigma^{i}(0 \leqslant i \leqslant l-1)$. Then, the characters $\chi_{k}$ are induced from $\sigma_{k}$ and $\sigma_{l-k}$, namely,

$$
\chi_{k}=\operatorname{Ind} \sigma_{k}=\operatorname{Ind} \sigma_{l-k}
$$

for all $k \in\left\{1, \cdots, \frac{l-1}{2}\right\}$. 
Fix an embedding $\overline{\mathbb{Q}}^{\times} \hookrightarrow \overline{\mathbb{Q}}_{p}^{\times}$and regard any character as $p$-adic one. Let $\operatorname{Irr}\left(D_{2 l}\right)$ be the set of all irreducible characters of $D_{2 l}$. For $\chi \in \operatorname{Irr}\left(D_{2 l}\right)$, put $\mathscr{O}_{\chi}=\mathbb{Z}_{p}[\operatorname{Im} \chi]$ and define

$$
e_{\chi}=\frac{\chi(1)}{2 l} \sum_{g \in D_{2 l}} \chi\left(g^{-1}\right) g \in \mathscr{O}_{\chi}\left[D_{2 l}\right]
$$

Let $M$ be a module over $\mathbb{Z}_{p}\left[D_{2 l}\right]$. We call $e_{\chi}\left(M \otimes \mathscr{O}_{\chi}\right)$ the $\chi$-part of $M$ and simply denote this by $M^{\chi}$. Put $\mathscr{O}=\mathbb{Z}_{p}\left[\zeta_{l}\right]$. Since $\left\{e_{\chi}\right\}_{\chi \in \operatorname{Irr}\left(D_{2 l}\right)}$ is orthogonal idempotents of $\mathscr{O}\left[D_{2 l}\right]$ and $1_{\mathscr{O}\left[D_{2 l}\right]}=\sum_{\chi \in \operatorname{Irr}\left(D_{2 l}\right)} e_{\chi}$, we may write

$$
M \otimes \mathscr{O}=\bigoplus_{\chi \in \operatorname{Irr}\left(D_{2 l}\right)} \tilde{M}^{\chi}
$$

where $\tilde{M}^{\chi}=e_{\chi}(M \otimes \mathscr{O})$. On the other hand, it is well-known that

$$
M \otimes \mathscr{O}=\bigoplus_{i=0}^{l-1} M^{\sigma_{i}}
$$

as an $\mathscr{O}[\langle a\rangle]$-module where $M^{\sigma_{i}}=\left\{x \in M \otimes \mathscr{O} \mid a x=\sigma_{i}(a) x\right\}$. In particular, when $M$ is finite, we have

$$
\# \bigoplus_{k=1}^{\frac{l-1}{2}} \tilde{M}^{\chi_{k}}=\frac{\#(M \otimes \mathscr{O})}{\#\left(\tilde{M}^{\varepsilon} \oplus \tilde{M}^{\eta}\right)}=\frac{\#(M \otimes \mathscr{O})}{\# M^{\sigma_{0}}}=\# \bigoplus_{k=1}^{l-1} M^{\sigma_{k}}
$$

since $\tilde{M}^{\varepsilon} \oplus \tilde{M}^{\eta}=\{x \in M \otimes \mathscr{O} \mid a x=x\}=M^{\sigma_{0}}$.

Lemma 2.1. Let $d_{k}=\left[\mathscr{O}: \mathscr{O}_{\chi_{k}}\right]$. If $M$ is a finite $\mathbb{Z}_{p}\left[D_{2 l}\right]$-module, then

$$
\left(\# M^{\chi_{k}}\right)^{d_{k}}=\left(\# M^{\sigma_{k}}\right)^{2}
$$

for all $k \in\left\{1, \cdots, \frac{l-1}{2}\right\}$.

Proof. Since $e_{\chi_{k}}=e_{\sigma_{k}}+e_{\sigma_{l-k}}$ in $\mathscr{O}\left[D_{2 l}\right]$, we have the natural homomorphism

$$
f: \tilde{M}^{\chi_{k}} \longrightarrow M^{\sigma_{k}} \oplus M^{\sigma_{l-k}}, \quad e_{\chi_{k}} x \mapsto\left(e_{\sigma_{k}} x, e_{\sigma_{l-k}} x\right)
$$

as abelian groups. Take $x \in M \otimes \mathscr{O}$ with $\left(e_{\sigma_{k}} x, e_{\sigma_{l-k}} x\right)=(0,0)$. This yields $e_{\chi_{k}} x=e_{\sigma_{k}} x+e_{\sigma_{l-k}} x=0$, which implies that $f$ is injective. Thus the equation (2.2) leads to

$$
\# \tilde{M}^{\chi_{k}}=\#\left(M^{\sigma_{k}} \oplus M^{\sigma_{l-k}}\right)
$$

for each $k$, therefore $f$ is also surjective. Note that $b e_{\sigma_{k}}=e_{\sigma_{l-k}} b$ and $b e_{\sigma_{l-k}}=e_{\sigma_{k}} b$. The homomorphism

$$
M^{\sigma_{k}} \longrightarrow M^{\sigma_{l-k}}, \quad x \mapsto b x
$$


is an isomorphism because

$$
M^{\sigma_{l-k}} \longrightarrow M^{\sigma_{k}}, \quad x \mapsto b x
$$

is its inverse map. It follows that $\# M^{\sigma_{k}}=\# M^{\sigma_{l-k}}$, so $\# \tilde{M}^{\chi_{k}}=\left(\# M^{\sigma_{k}}\right)^{2}$. On the other hand, we know $\# \tilde{M}^{\chi_{k}}=\left(\# M^{\chi_{k}}\right)^{d_{k}}$ by

$$
M \otimes \mathscr{O} \simeq M \otimes\left(\mathscr{O}_{\chi_{k}}^{d_{k}}\right) \simeq\left(M \otimes \mathscr{O}_{\chi_{k}}\right)^{d_{k}}
$$

as $\mathscr{O}_{\chi_{k}}\left[D_{2 l}\right]$-modules. This completes the proof.

Now we give a proof of Theorem 1.1. In the following arguments, we identify $\operatorname{Gal}\left(L^{+} / F^{+}\right)$with $D_{2 l}=\langle a, b\rangle$. Let $K^{+}$be the fixed field of $\langle a\rangle$ in $L^{+}$and $K=K^{+}(\sqrt{-r})$. For an irreducible character $\psi$ of $\operatorname{Gal}\left(L^{+} / F^{+}\right)$, we define the characters $\psi^{+}$and $\psi^{-}$of $\operatorname{Gal}\left(L / F^{+}\right)$by

$$
\psi^{+}(g)=\psi\left(\left.g\right|_{L^{+}}\right), \quad \psi^{-}(g)=\gamma\left(\left.g\right|_{F}\right) \psi\left(\left.g\right|_{L^{+}}\right),
$$

respectively, where $\gamma$ is the non-trivial character of $\operatorname{Gal}\left(F / F^{+}\right)$. In fact, we know that $\psi^{+}$is even while $\psi^{-}$is odd. For a character $\sigma$ of $\operatorname{Gal}\left(L^{+} / K^{+}\right)$, define the characters $\sigma^{ \pm}$of $\operatorname{Gal}\left(L / K^{+}\right)$in the same manner. Using these notations and Theorem 4.21 of [2], we obtain

$$
\operatorname{Irr}\left(\operatorname{Gal}\left(L / F^{+}\right)\right)=\left\{\varepsilon^{ \pm}, \eta^{ \pm}, \chi_{1}^{ \pm}, \cdots, \chi_{\frac{l-1}{2}}^{ \pm}\right\}
$$

and

$$
\operatorname{Hom}\left(\operatorname{Gal}\left(L / K^{+}\right), \overline{\mathbb{Q}}_{p} \times\right)=\left\{\sigma_{0}^{ \pm}, \cdots, \sigma_{l-1}^{ \pm}\right\} .
$$

First, we treat the characters of two-dimensional representations. For a finite $\mathbb{Z}_{p}\left[\operatorname{Gal}\left(L / F^{+}\right)\right]$-module $M$, we have

$$
\left(\# M^{\chi_{k}^{ \pm}}\right)^{d_{\sigma_{k}^{ \pm}} / d_{\chi_{k}^{ \pm}}}=\left(\# M^{\sigma_{k}^{ \pm}}\right)^{2}
$$

by Lemma 2.1, and therefore

$$
\frac{\left(\# H^{2}\left(O_{L}^{\prime}, \mathbb{Z}_{p}(n)\right)^{\chi_{k}^{ \pm}}\right)^{d_{\sigma_{k}^{ \pm}} / d_{\chi_{k}^{ \pm}}}}{\left(\# H^{1}\left(O_{L}^{\prime}, \mathbb{Z}_{p}(n)\right)^{\chi_{k}^{ \pm}}\right)^{d_{\sigma_{k}^{ \pm}} / d_{\chi_{k}^{ \pm}}}}=\frac{\left(\# H^{2}\left(O_{L}^{\prime}, \mathbb{Z}_{p}(n)\right)^{\sigma_{k}^{ \pm}}\right)^{2}}{\left(\# H^{1}\left(O_{L}^{\prime}, \mathbb{Z}_{p}(n)\right)^{\sigma_{k}^{ \pm}}\right)^{2}}
$$

We remark that characters of dihedral groups take real values. Since $\overline{\chi_{k}^{ \pm}}=\chi_{k}^{ \pm}=$ Ind $\left(\sigma_{k}^{ \pm}\right)^{-1}$ by (2.1), it follows from Chapter VII, Proposition 10.4 (iv) of [5] that

$$
\mathscr{L}\left(L / F^{+}, \overline{\chi_{k}^{ \pm}}, 1-n\right)=\mathscr{L}\left(L / K^{+},\left(\sigma_{k}^{ \pm}\right)^{-1}, 1-n\right) .
$$


By the way, we can apply Theorem 1.2 to $L / K^{+}$because $\operatorname{Gal}\left(L / K^{+}\right)$is the direct product of two cyclic groups of order $l$ and 2 . Hence,

$$
\mathscr{L}\left(L / K^{+},\left(\sigma_{k}^{(n)}\right)^{-1}, 1-n\right)^{d_{\sigma_{k}^{(n)}}} \sim_{p} \frac{\# H^{2}\left(O_{L}^{\prime}, \mathbb{Z}_{p}(n)\right)^{\sigma_{k}^{(n)}}}{\# H^{1}\left(O_{L}^{\prime}, \mathbb{Z}_{p}(n)\right)^{\sigma_{k}^{(n)}}}
$$

where $\sigma_{k}^{(n)}=\sigma_{k}^{+}$if $n$ is even and $\sigma_{k}^{(n)}=\sigma_{k}^{-}$if $n$ is odd. Since $\chi_{k}^{ \pm}(1)=2$, the relationship (2.5) is equivalent to

$$
\mathscr{L}\left(L / K^{+},\left(\sigma_{k}^{(n)}\right)^{-1}, 1-n\right)^{\chi_{k}^{(n)}(1) \cdot d_{\sigma_{k}^{(n)}}} \sim_{p} \frac{\left(\# H^{2}\left(O_{L}^{\prime}, \mathbb{Z}_{p}(n)\right)^{\sigma_{k}^{(n)}}\right)^{2}}{\left(\# H^{1}\left(O_{L}^{\prime}, \mathbb{Z}_{p}(n)\right)^{\sigma_{k}^{(n)}}\right)^{2}} .
$$

Combining this with (2.3) and (2.4), we deduce that

$$
\mathscr{L}\left(L / F^{+}, \overline{\chi_{k}^{(n)}}, 1-n\right)^{\chi_{k}^{(n)}(1) \cdot d_{\sigma_{k}^{(n)}}} \sim_{p} \frac{\left(\# H^{2}\left(O_{L}^{\prime}, \mathbb{Z}_{p}(n)\right)^{\chi_{k}^{(n)}}\right)^{d_{\sigma_{k}^{(n)}} / d_{\chi_{k}(n)}}}{\left(\# H^{1}\left(O_{L}^{\prime}, \mathbb{Z}_{p}(n)\right)^{\chi_{k}^{(n)}}\right)^{d_{\sigma_{k}^{(n)}} / d_{\chi_{k}^{(n)}}}}
$$

i.e.

$$
\mathscr{L}\left(L / F^{+}, \overline{\chi_{k}^{(n)}}, 1-n\right)^{\chi_{k}^{(n)}(1) \cdot d_{\chi_{k}^{(n)}}} \sim_{p} \frac{\# H^{2}\left(O_{L}^{\prime}, \mathbb{Z}_{p}(n)\right)^{\chi_{k}^{(n)}}}{\# H^{1}\left(O_{L}^{\prime}, \mathbb{Z}_{p}(n)\right)^{\chi_{k}^{(n)}}}
$$

This completes the proof for the case $\chi=\chi_{k}^{ \pm}$.

We next explain the cases $\chi=\varepsilon^{ \pm}$that are linear characters. For this purpose we prepare the following lemma, which seems folklore for experts.

Lemma 2.2. Let $L / K$ be a finite Galois extension of number fields and suppose $p$ is prime to $[L: K]$. Then the canonical homomorphism

$$
H^{i}\left(O_{K}^{\prime}, \mathbb{Z}_{p}(n)\right) \longrightarrow H^{i}\left(O_{L}^{\prime}, \mathbb{Z}_{p}(n)\right)^{\operatorname{Gal}(L / K)}
$$

is bijective for any $i$ and any $n$.

Proof. We write $A=O_{K}[1 / p], B=O_{L}[1 / p]$ and $\Gamma=\operatorname{Gal}(L / K)$. Let $\mu_{p^{r}}$ denote the group scheme of $p^{r}$-th root of unity over $A$. Then $\mu_{p^{r}}$ is étale and finite over $A$ since $p$ is invertible in $A$, and the Tate twist $\mu_{p^{r}}^{\otimes n}$ is also representable by an étale finite group scheme over $A$. Put $G=\mu_{p^{r}}^{\otimes n}$ and let $\operatorname{Res}_{B / A} G$ denote the Weil restriction with respect to the finite extension $B / A$. We have the natural inclusion $\iota: G \rightarrow \operatorname{Res}_{B / A} G$ and the natural norm homomorphism $\mathrm{Nr}: \operatorname{Res}_{B / A} G \rightarrow G$. Furthermore, it is readily seen that

(1) $\mathrm{Nr} \circ \iota$ is equal to the multiplication-by- $[L: K]$ map over $G$;

(2) $\iota \circ \mathrm{Nr}$ is equal to $\sum_{\gamma \in \Gamma} \gamma$ over $\operatorname{Res}_{B / A} G$. 
Note that the Weil restriction is nothing but the direct image of the étale sheaf on $\operatorname{Spec} B$ by the morphism $\pi: \operatorname{Spec} B \rightarrow \operatorname{Spec} A$. Therefore, the canonical homomorphism

$$
H^{i}\left(A, \operatorname{Res}_{B / A} G\right) \longrightarrow H^{i}(B, G)
$$

is bijective since $\pi$ is finite (cf. [1], Expo VIII, Cor 5.6). Moreover, the homomorphism $\iota: G \rightarrow \operatorname{Res}_{B / A} G$ gives rise to a homomorphism

$$
\iota: H^{i}(A, G) \longrightarrow H^{i}\left(A, \operatorname{Res}_{B / A} G\right) \simeq H^{i}(B, G),
$$

which is nothing but the homomorphism induced by $\pi: \operatorname{Spec} B \rightarrow \operatorname{Spec} A$. On the other hand, $\mathrm{Nr}: \operatorname{Res}_{B / A} G \rightarrow G$ gives rise to a homomorphism

$$
\mathrm{Nr}: H^{i}(B, G) \simeq H^{i}\left(A, \operatorname{Res}_{B / A} G\right) \longrightarrow H^{i}(A, G)
$$

It follows from (1) and (2) that

(1)' $\mathrm{Nr} \circ \iota$ is equal to the multiplication-by- $[L: K]$ map over $H^{i}(A, G)$;

(2)' $\iota \circ \mathrm{Nr}$ is equal to $\sum_{\gamma \in \Gamma} \gamma$ over $H^{i}(B, G)$.

Passing to the limit, we obtain homomorphisms

$$
\iota: H^{i}\left(A, \mathbb{Z}_{p}(n)\right) \longrightarrow H^{i}\left(B, \mathbb{Z}_{p}(n)\right)
$$

and

$$
\mathrm{Nr}: H^{i}\left(B, \mathbb{Z}_{p}(n)\right) \longrightarrow H^{i}\left(A, \mathbb{Z}_{p}(n)\right)
$$

It follows again from (1)' and (2)' that

(1)" $\mathrm{Nr} \circ \iota$ is equal to the multiplication-by- $[L: K]$ map over $H^{i}(A, \mathbb{Z}(n))$;

(2)" $\iota \circ \mathrm{Nr}$ is equal to $\sum_{\gamma \in \Gamma} \gamma$ over $H^{i}(B, \mathbb{Z}(n))$,

and therefore $\iota \circ \mathrm{Nr}$ is equal to the multiplication-by- $[L: K]$ map over $H^{i}\left(B, \mathbb{Z}_{p}(n)\right)^{\Gamma}$. Note that the two multiplication-by- $[L: K]$ maps $\mathrm{Nr} \circ \iota: H^{i}\left(A, \mathbb{Z}_{p}(n)\right) \rightarrow$ $H^{i}\left(A, \mathbb{Z}_{p}(n)\right)$ and $\iota \circ \mathrm{Nr}: H^{i}\left(B, \mathbb{Z}_{p}(n)\right)^{\Gamma} \rightarrow H^{i}\left(B, \mathbb{Z}_{p}(n)\right)^{\Gamma}$ are bijective because $p$ does not divide $[L: K]$. This implies that $\iota: H^{i}\left(A, \mathbb{Z}_{p}(n)\right) \rightarrow H^{i}\left(B, \mathbb{Z}_{p}(n)\right)^{\Gamma}$ is bijective.

Let $\gamma^{+}: \operatorname{Gal}\left(F / F^{+}\right) \rightarrow{\overline{\mathbb{Q}_{p}}}^{\times}$and $\gamma^{-}: \operatorname{Gal}\left(F / F^{+}\right) \rightarrow{\overline{\mathbb{Q}_{p}}}^{\times}$be the trivial and non-trivial character, respectively. Note that $d_{\gamma^{ \pm}}=1,\left(\gamma^{ \pm}\right)^{-1}=\gamma^{ \pm}$, and $\overline{\varepsilon^{ \pm}}=\varepsilon^{ \pm}$. We can apply Theorem 1.2 to the quadratic extension $F / F^{+}$, so,

$$
\mathscr{L}\left(F / F^{+},\left(\gamma^{(n)}\right)^{-1}, 1-n\right) \sim_{p} \frac{\# H^{2}\left(O_{F}^{\prime}, \mathbb{Z}_{p}(n)\right)^{\gamma^{(n)}}}{\# H^{1}\left(O_{F}^{\prime}, \mathbb{Z}_{p}(n)\right)^{\gamma^{(n)}}} .
$$

For the left side of (2.6), it follows from Chapter VII, Proposition 10.4 (iii) of [5] that

$$
\mathscr{L}\left(L / F^{+}, \overline{\varepsilon^{ \pm}}, 1-n\right)=\mathscr{L}\left(F / F^{+},\left(\gamma^{ \pm}\right)^{-1}, 1-n\right) .
$$


Since $g e_{\varepsilon^{ \pm}}=e_{\varepsilon^{ \pm}}$for all $g \in \operatorname{Gal}(L / F)$, we find

$$
\begin{aligned}
& H^{i}\left(O_{F}^{\prime}, \mathbb{Z}_{p}(n)\right)^{\gamma^{+}} \oplus H^{i}\left(O_{F}^{\prime}, \mathbb{Z}_{p}(n)\right)^{\gamma^{-}} \simeq H^{i}\left(O_{F}^{\prime}, \mathbb{Z}_{p}(n)\right) \\
& \simeq H^{i}\left(O_{L}^{\prime}, \mathbb{Z}_{p}(n)\right)^{\operatorname{Gal}(L / F)} \\
& \simeq H^{i}\left(O_{L}^{\prime}, \mathbb{Z}_{p}(n)\right)^{\varepsilon^{+}} \oplus H^{i}\left(O_{L}^{\prime}, \mathbb{Z}_{p}(n)\right)^{\varepsilon^{-}}
\end{aligned}
$$

and

$$
\begin{aligned}
H^{i}\left(O_{F}^{\prime}, \mathbb{Z}_{p}(n)\right)^{\gamma^{+}} & \simeq H^{i}\left(O_{F}^{\prime}, \mathbb{Z}_{p}(n)\right)^{\operatorname{Gal}\left(F / F^{+}\right)} \\
& \simeq H^{i}\left(O_{F}^{\prime}, \mathbb{Z}_{p}(n)\right) \\
& \simeq H^{i}\left(O_{L}^{\prime}, \mathbb{Z}_{p}(n)\right)^{\operatorname{Gal}\left(L / F^{+}\right)} \\
& \simeq H^{i}\left(O_{L}^{\prime}, \mathbb{Z}_{p}(n)\right)^{\varepsilon^{+}}
\end{aligned}
$$

by Lemma 2.2. Thus, the following equations

$$
\# H^{i}\left(O_{F}^{\prime}, \mathbb{Z}_{p}(n)\right)^{\gamma^{ \pm}}=\# H^{i}\left(O_{L}^{\prime}, \mathbb{Z}_{p}(n)\right)^{\varepsilon^{ \pm}}
$$

hold for $i=1,2$. These (2.6), (2.7) and (2.8) lead to

$$
\mathscr{L}\left(L / F^{+}, \overline{\varepsilon^{(n)}}, 1-n\right) \sim_{p} \frac{\# H^{2}\left(O_{L}^{\prime}, \mathbb{Z}_{p}(n)\right)^{\varepsilon^{(n)}}}{\# H^{1}\left(O_{L}^{\prime}, \mathbb{Z}_{p}(n)\right)^{\varepsilon^{(n)}}} .
$$

This completes the proof for the case $\chi=\varepsilon^{ \pm}$.

Similarly, by [5, Proposition 10.4 (iii) in Ch. VII], we can apply Theorem 1.2 to $K / F^{+}$to obtain the desired result for the case $\chi=\eta^{ \pm}$.

Acknowledgments. The author would like to thank Professors Shōichi Nakajima, Shin Nakano, Yoshitaka Hachimori and Yoshichika Iizuka for their valuable advice and encouragement. He also thanks Professor Noriyuki Suwa, who communicated the proof of Lemma 2.2.

\section{References}

[1] A. Grothendieck et al, Théorie des Topos et Cohomologie Etale des Schémas, Lecture Notes in Mathmatics, vol. 270, 1972.

[2] I. Martin Isaacs, Character Theory of Finite Groups, Pure and Applied Mathematics 69, Academic Press, 1976.

[3] M. Kolster, T. Nguyen Quang Do, V. Fleckinger, Twisted S-Units, p-adic class number formulas, and the Lichtenbaum conjectures, Duke Mathematical Journal 84 (1996), no.3, 679-717.

[4] Y. Konomi, The ideal class groups of dihedral extensions over imaginary quadratic fields and the special values of the Artin L-function, J. Number Theory 131 (2011), no.6, 1062-1069. 
[5] J. Neukirch, Algebraic Number Theory, GdmW., vol. 322, Springer, 1999.

[6] V.P. Snaith, Algebraic K-groups as Galois Modules, Progress in Mathematics 206, Birkhäuser, 2002.

[7] V. Voevodsky, On motivic cohomology with $\mathbb{Z} /$ l-coefficients, Annals of Mathematics 174 (2011), 401-438.

[8] C. Weibel, 2007 Trieste lectures on the proof of the Bloch-Kato conjecture, some recent developments in algebraic $K$-theory, ICTP Lecture Notes $\mathbf{2 3}$ (Abdus Salam International Centre for Theoretical Physics, Trieste, 2008), 277-305.

[9] A. Wiles, On a conjecture of Brumer, Annals of Mathematics 131 (1990), $555-565$.

Address: Yutaka Konomi: Department of Mathematics, Gakushuin University, Mejiro, Toshima-ku, Tokyo, 171-8588 Japan.

E-mail: konomi@math.gakushuin.ac.jp

Received: 13 September 2013; revised: 18 September 2014 